\title{
A ESTIMATIVA DA CONTRIBUIÇÃO DE PERNAS NO NADO DE CRAWL
}

\author{
ESTIMATED CONTRIBUITION OF LEGS IN THE CRAWL SWIMMING
}

\section{CONTRIBUCIÓN ESTIMADA DE LAS PIERNAS EN EL CROL}

\author{
Raísa Valvassori \\ https://orcid.org/0000-0003-2925-8163 (ib \\ http://lattes.cnpq.br/1977050518227147 \\ Universidade de São Paulo (São Paulo, SP - Brasil) \\ raisavalvassori@gmail.com \\ Rudolf Huebner \\ https://orcid.org/0000-0003-2613-304X (i) \\ http://lattes.cnpq.br/9514309218273598 \\ Universidade Federal de Minas Gerais (Belo Horizonte, MG - Brasil) \\ rudolf@ufmg.br \\ João Paulo Vilas-Boas \\ https://orcid.org/0000-0002-4109-2939 (D) \\ Universidade do Porto (Porto, PT - Portugal) \\ jpvb@fade.up.pt \\ Alberto Carlos Amadio \\ https://orcid.org/0000-0002-2527-2163 id \\ http://lattes.cnpq.br/0634221030546752 8 \\ Universidade de São Paulo (São Paulo, SP - Brasil) \\ acamadio@usp.br \\ Júlio Cerca Serrão \\ https://orcid.org/0000-0002-3646-3387 (iD \\ http://lattes.cnpq.br/9124685212860479 9 \\ Universidade de São Paulo (São Paulo, SP - Brasil) \\ jcserrao@usp.br \\ Bruno Mezêncio \\ https://orcid.org/0000-0001-6293-5571 \\ http://lattes.cnpq.br/9793275013451101 \\ Universidade de São Paulo (São Paulo, SP - Brasil) \\ mezencio@usp.br
}

\section{Resumo}

O objetivo do estudo foi avaliar a contribuição relativa de membros inferiores no nado crawl utilizando o método de perturbação da velocidade (VPM) em atletas de diferentes níveis de habilidade. Participaram do estudo 106 nadadores, os quais foram divididos igualmente em dois grupos de acordo com o nível de habilidade (competitivo e recreativo). Os nadadores realizaram 8 tiros máximos de 15 metros nadando crawl em 4 diferentes condições com o mínimo de 2'30" de intervalo entre os tiros (nado completo; completo com paraquedas; apenas pernada e; pernada com paraquedas). Todos os tiros foram filmados com uma câmera subaquática (GoPro Hero4 Black), e os parâmetros de interesse registrados foram: velocidade média do nadador; o arrasto ativo e potência de nado em cada uma das condições. Para comparação estatística da contribuição relativa de membros inferiores entre os 
grupos foi realizado um teste T. O nível de significância adotado foi de $p<0.05$. O resultado principal mostrou na comparação entre grupos que a contribuição relativa de membros inferiores, foram significativamente maiores para o grupo recreativo, $33,9 \pm 23,1 \%$ vs. $22,5 \pm 10,3 \%(p=0.02)$. Por fim, o VPM se mostrou uma técnica de fácil execução capaz de avaliar a contribuição relativa de membros inferiores no nado crawl, tornando esta informação acessível para treinadores.

Palavras-chave: Método de Perturbação de Velocidade (VPM); Nado Crawl; Propulsão de Pernas.

\section{Abstract}

The aim of the study was to evaluate the relative contribution of lower limbs in the crawl swim using the velocity disturbance method (VPM) in athletes of different skill levels. 106 swimmers participated in the study, they were equally divided into two groups according to their skill level (competitive and recreational). The swimmers performed 8 maximum sprints of 15 meters by swimming crawl in 4 different conditions with a minimum of 2'30" between sprints (full swim; complete with parachute; only kick and; kick with parachute). All sprints were filmed with an underwater camera (GoPro Hero4 Black), and the parameters of interest recorded were: average swimmer speed, active drag and swimming power in each of the conditions. For statistical comparison of the relative contribution of lower limbs between groups a test-T was performed. The significance level adopted was $p<0.05$. The main result showed in the comparison between groups that the relative contribution of lower limbs, were significantly higher for the recreational group, $33.9 \pm 23.1 \%$ vs. $22.5 \pm 10.3 \%(p=0.02)$. Finally, the VPM proved to be an easy to perform technique capable of assessing the relative contribution of lower limbs in the crawl swim, making this information accessible to coaches.

Keywords: Velocity Disturbance Method (VPM); Crawl Swim; Leg Propulsion.

\section{Resumen}

El objetivo del estudio fue evaluar la contribución relativa de los miembros inferiores en el nado de arrastre utilizando el método de alteración de la velocidad (VPM) en atletas de diferentes niveles de habilidad. 106 nadadores participaron en el estudio, se dividieron igualmente en dos grupos según su nivel de habilidad (competitivo y recreativo). Los nadadores realizaron 8 sprints máximos de 15 metros mediante crol de nado en 4 condiciones diferentes con un mínimo de 2'30 "entre sprints (nado completo; completo con paracaídas; solo patada y; patada con paracaídas). Todos los sprints fueron filmados con un cámara (GoPro Hero4 Black), y los parámetros de interés registrados fueron: velocidad media del nadador, resistencia activa y potencia de nado en cada una de las condiciones. Para la comparación estadística de la contribución relativa de las extremidades inferiores entre grupos, se realizó un test-T. El nivel de significancia adoptado fue $\mathrm{p}<0.05$. El principal resultado mostró en la comparación entre grupos que la contribución relativa de miembros inferiores, fue significativamente mayor para el grupo recreativo, $33.9 \pm 23.1 \%$ vs. $22.5 \pm 10.3 \%(p=0.02)$, el VPM demostró ser una técnica fácil de realizar capaz de evaluar la contribución relativa de las extremidades inferiores en el crol, haciendo que esta información sea accesible a los entrenadores.

Palabras clave: Método de Perturbación de la Velocidad (VPM); Nado Crol; Propulsión de la Pierna.

\section{INTRODUÇÃO}

A determinação da contribuição relativa de membros superiores e inferiores no nado crawl é um tema de interesse na literatura cientifica. Uma das técnicas utilizadas para esta análise é a comparação da velocidade de nado (BUCHER, 1974; KARPOVICH, 1935). Bucher (1974) instruiu os nadadores a realizar tiros máximos em nado completo, utilizando apenas ação de braços e utilizando apenas ações de pernas. Foi verificado que nadadores iniciante e profissionais apresentam em média a mesma proporção de contribuição relativa entre braços ( $~ 90 \%$ da velocidade desenvolvida em nado completo) e pernas ( $60 \%$ da velocidade desenvolvida em nado completo). No entanto comparando dois nadadores de elite da amostra que apresentaram desempenho semelhante no nado completo foi observada uma grande 
diferença entre a utilização das ações de braços e pernas. Ambos tiveram um tempo de 8,7 s no tiro de $15 \mathrm{~m}$ crawl nado completo, porém no tiro de apenas pernas os resultados foram 12,1 e 12,8 s, enquanto para ação de braços os tempos foram de 10,2 e 9,3 s (BUCHER, 1974).

Este método apresenta a óbvia limitação de que a soma das contribuições relativas extrapola os $100 \%$, isso é esperado pela variação das condições de escoamento e pela relação não linear entre arrasto e velocidade. Karpovich (1935) foi o primeiro a identificar esta distorção e sugeriu que a proporção entre as velocidades deveria seguir aquela de um triangulo retângulo (Equação 1), esta proporção foi estabelecida baseando-se na relação quadrática entre arrasto e velocidade.

$$
V_{\text {completo }}^{2}=V_{\text {perna }}^{2}+V_{\text {braço }}^{2}
$$

Considerando que as velocidades obtidas nos testes se relacionam à força aplicada como $F=0,65 V^{2.05}$, a contribuição relativa das pernas foi estimada entre 23 e $30 \%$ da força total produzida (KARPOVICH, 1935). Esta equação foi determinada de forma subjetiva pelo autor, baseando-se na conhecida relação quadrática entre arrasto e velocidade no meio fluido (AMAR, 1920), porém com uma correção dos coeficientes por considerar que seus nadadores eram grandes, sendo esta uma limitação desta abordagem. Também foi verificado neste estudo que a contribuição de pernas era maior em indivíduos de melhor desempenho.

Uma técnica experimental utilizada para avaliar contribuição relativa de membros inferiores é a técnica de rebocamento. Nesta técnica, os nadadores eram rebocados em velocidades constantes realizando uma ação máxima de pernas. Os testes eram realizados em velocidades progressivas e foi observado que a partir da velocidade de $1.5 \mathrm{~m} / \mathrm{s}$ o cabo se mantinha estendido durante o teste, indicando que a partir desta velocidade os membros inferiores não mais poderiam contribuir para a velocidade de nado. Valores menores de até $1.2 \mathrm{~m} / \mathrm{s}$ foram observados para nadadores menos habilidosos (COUNSILMAN, 1971).

Outra técnica experimental utilizada para este objetivo foi a utilização do MAD system (sistema de mensuração do arrasto ativo). O sistema consiste em uma série de anteparos, onde o nadador deve realizar as braçadas apoiando a mão nestes anteparos e não pode realizar ações de pernas (HOLLANDER et al., 1986). Como apenas a força propulsiva de membros superiores é registrada no MAD system, este método foi utilizado para determinar a potência adicional fornecida pela ação de membros inferiores. Para isto inicialmente foi 
determinada a função que caracterizava o arrasto de cada voluntário em função da velocidade e calculada a potência máximo utilizando apenas os braços. Posteriormente eles nadaram em máxima velocidade utilizando o MAD, mas com ação de pernas. A velocidade foi registrada, o arrasto foi estimado pela função obtida previamente e a diferença na potência entre as situações ( 11\%) foi atribuída à ação de pernas. No entanto como o MAD system melhora a eficiência da ação de braços devido aos anteparos utilizados, acredita-se que esta contribuição possa ser ainda maior (HOLLANDER et al., 1988).

Porém estas técnicas experimentais são de difícil aplicação, enquanto os métodos baseados apenas na velocidade têm limitações severas devido à relação não linear entre propulsão e velocidade. No entanto, existe um método experimental mais simples para determinação do arrasto ativo, e consequentemente da potência de nado, que permite uma fácil avaliação da contribuição relativa dos membros no nado, o método de perturbação da velocidade, conhecido como VPM (KOLMOGOROV; DUPLISHCHEVA, 1992).

Este método utiliza a adição de um corpo hidrodinâmico de arrasto conhecido para avaliação do arrasto. Neste método o nadador deve executar dois tiros máximos, sendo um deles rebocando o corpo hidrodinâmico e o outro em nado livre. A velocidade média é mensurada em ambos os testes e são assumidos dois pressupostos teóricos para possibilitar a estimativa do arrasto ativo: i) o nadador produz a mesma potência durante os dois testes e; ii) a relação entre velocidade e arrasto é quadrática (KOLMOGOROV; DUPLISHCHEVA, 1992).

Utilizando esta técnica seria possível identificar com facilidade a contribuição relativa de membros em nadadores, o que pode ser uma valiosa informação de controle e/ou planejamento para treinadores. Desta forma, o objetivo do estudo foi avaliar a contribuição relativa de membros inferiores no nado crawl utilizando o VPM em atletas de diferentes níveis de habilidade.

\section{MÉTODOS}

A amostra foi composta por 106 nadadores (Idade: 22,6 \pm 9.2 anos; Altura: $1.68 \pm$ $0.09 \mathrm{~m}$; Massa corporal: $66.36 \pm 13.03 \mathrm{Kg}$ ), sendo 48 mulheres e 58 homens. Destes 53 praticavam natação de forma não competitiva, denominado grupo recreativo, e 53 treinavam ou treinaram natação com finalidade competitiva, tendo disputado o campeonato brasileiro nos últimos 12 meses (Desempenho: $78.7 \pm 5.84 \%$ do recorde mundial dos 50m nado livre), 
denominado grupo competitivo. Todos os procedimentos da pesquisa foram aprovados pelo Comitê de Ética em Pesquisa da Universidade (Processo 26478013.0.0000.5391).

Inicialmente os participantes responderam a uma anamnese a respeito de sua experiência pregressa em natação. Na sequência os participantes realizavam o aquecimento e a familiarização com os equipamentos. A coleta de dados consistiu em 8 tiros máximos de 15 metros nadando crawl em 4 diferentes condições: nado completo; completo com paraquedas; apenas pernada e; pernada com paraquedas. Nos testes onde os voluntários utilizaram apenas a pernada de crawl, eles deveriam manter os braços estendidos em prolongamento à cabeça. Todos os tiros foram filmados com uma câmera subaquática para análise e cálculo dos parâmetros de interesse: a velocidade média do nadador; o arrasto ativo e a potência de nado em cada condição.

Os testes foram realizados na piscina da Escola de Educação Física e Esporte da Universidade de São Paulo e nas piscinas dos clubes que participaram da pesquisa. A água sempre foi mantida entre 25 e $28^{\circ} \mathrm{C}$. Os testes foram realizados na segunda raia da piscina e os voluntários foram orientados a nadar sobre a marcação da raia no fundo da piscina. A corda da raia 1 foi removida para os testes com o objetivo de melhorar a visualização dos movimentos dos nadadores, a corda da raia 2 foi mantida na piscina.

Antes da realização dos oito tiros, foi solicitado aos participantes que realizassem um aquecimento livre, de forma que eles se sentissem aptos a realizar os esforços máximos que os testes requeriam. Não foi adotado um tempo padrão de aquecimento devido às grandes variações de condicionamento físico esperadas na amostra. O tempo utilizado pelos participantes variou entre 10 e 25 minutos, sendo que os praticantes de natação competitiva foram aqueles que executaram um aquecimento de maior duração.

Após o aquecimento os indivíduos realizavam uma familiarização com paraquedas. Os voluntários deveriam nadar pelo menos um trajeto de $15 \mathrm{~m}$ com o paraquedas, porém o voluntário poderia realizar quantas tentativas de familiarização quisesse. Foram utilizadas entre uma e quatro tentativas pelos voluntários do estudo, sendo que 70,1\% dos voluntários relataram já ter experiência prévia com a utilização de paraquedas.

Aproximadamente três minutos após a realização da familiarização, tinha início a coleta de dados. Os oito tiros de 15 metros em nado crawl eram realizados em máximo esforço com no mínimo 2'30" de intervalo entre eles. 
Os voluntários foram instruídos a iniciar o teste com um impulso na parede, porém, eles já deveriam iniciar o teste na superfície da água e realizando o gesto específico daquele teste, desta forma, não foi permitida nenhuma fase submersa ou a utilização de ondulação na saída. Os voluntários também foram instruídos a não respirar durante o teste. Os dois tiros de cada condição sempre foram realizados seguidos, mas a ordem de execução das condições foi realizada de maneira aleatória.

Para avaliação cinemática do nado foi utilizada uma câmera GoPro Hero4 Black (sensor de 1/2,3" - 6,17 × 4,55 mm). A configuração de filmagem adotada foi 1080p wide (1920 x 1080, 16:9), com 119.88 fps. A opção de ajuste automático da luminosidade foi desabilitada. A câmera foi fixada a um tubo de PVC oco (110 mm de diâmetro) para permitir conexão wireless entre a câmera e um aparelho celular, desta forma os comandos para a câmera eram enviados pelo aparelho utilizando o aplicativo da própria câmera. Esta conexão também permitia a visualização on-line dos dados da câmera. Um operador iniciava a gravação por intermédio do celular 3 segundos antes do sinal de partida para os voluntários e terminava a gravação após o voluntário tocar a marca dos 15 metros. A câmera foi posicionada a uma profundidade de $35 \mathrm{~cm}$, distante $9,5 \mathrm{~m}$ da borda da piscina. Os testes foram realizados na segunda raia da piscina a uma distância de 3,5 metros da câmera, este posicionamento permitia um campo de visão de $6 \mathrm{~m}$, do $7^{\circ}$ ao $13^{\circ} \mathrm{m}$ da piscina. A velocidade média de cada tentativa foi calculada a partir do tempo gasto para o nadador cruzar a zona de análise, sendo considerada a cabeça do nadador o ponto de referência a cruzar os limites da zona de análise.

O arrasto ativo foi calculado pelo VPM (KOLMOGOROV; DUPLISHCHEVA, 1992) utilizando a equação 2 . Sendo $\mathrm{F}_{\mathrm{b}}$ a força de arrasto produzida pelo paraquedas, $\mathrm{v}_{2}$ a velocidade média do teste com paraquedas e $\mathrm{v}_{1}$ a velocidade média do nado livre.

$$
D=\frac{F_{b} v_{2} v_{1}^{2}}{v_{1}^{3}-v_{2}^{3}}
$$

O paraquedas utilizado possui área de superfície de $0.0144 \mathrm{~m}^{2}$ e a função do arrasto do paraquedas em relação à velocidade foi estimada previamente através de um modelo de regressão polinomial de terceira ordem aplicado nos dados de velocidade e força obtidos em um experimento de rebocamento do paraquedas a diferentes velocidades com uma célula de carga (Interface WMC - 450N) fixada ao conector do paraquedas. Este modelo 
de regressão apresentou uma soma dos quadrados dos erros (SSE) de 5.41 e $r^{2}$ de 0,99. A força média e a velocidade média de cada teste foram registradas e foram utilizadas para determinar o arrasto adicional. A partir dos resultados de arrasto de nado completo e apenas com ação de pernas também foram calculadas as potências de nado nas duas condições e a contribuição relativa de potência de nado com apenas membros inferiores em relação ao nado completo.

A normalidade dos dados foi verificada pelo teste de Kolmogorov-Smirnov e a igualdade de variância pelo teste de Levene.

Para comparação da velocidade, do arrasto ativo e da potência de nado entre as condições de nado completo ou apenas membros inferiores entre os dois grupos de desempenho, foi utilizada uma ANOVA fatorial com dois fatores e medidas repetidas sendo o primeiro fator a comparação principal (completo $\mathrm{x}$ membros inferiores) e o segundo fator o desempenho. Quando necessário foi utilizado o teste post-hoc de Student-Newman-Keuls (SNK). Para comparação da contribuição relativa de membros inferiores entre os grupos foi realizado um teste $T$. O nível de significância adotado foi de $p<0.05$ e as análises foram feitas no software SigmaStat 3.5 (Systat).

\section{RESULTADOS}

A tabela 1 apresenta os resultados das comparações de velocidade, arrasto ativo e potência de nado entre os grupos competitivo e recreativo e entre as condições de nado completo e apenas com ações de membros inferiores.

Tabela 1 - Resultados das comparações entre condições e grupos

\begin{tabular}{cccccccc}
\hline & \multicolumn{2}{c}{ Competitivo } & \multicolumn{2}{c}{ Recreativo } & \multicolumn{2}{c}{ p } & \\
\cline { 2 - 7 } & Completo & MMII & Completo & MMII & Grupo & Cond. & Int. \\
\hline Vel (m/s) & $1.74 \pm 0.14^{\mathrm{A}, \mathrm{B}}$ & $1.07 \pm 0.16^{\mathrm{B}}$ & $1.27 \pm 0.25^{\mathrm{A}}$ & $0.77 \pm 0.17$ & $<0.01$ & $<0.01$ & $<0.01$ \\
& & & $39.24 \pm$ & $19.21 \pm$ & & & \\
A (N) & $47.82 \pm 25.20^{\mathrm{A}, \mathrm{B}}$ & $15.11 \pm 5.37$ & $23.09^{\mathrm{A}}$ & 13.16 & $<0.01$ & $<0.01<0.01$ \\
& & & $51.31 \pm$ & & & & \\
& & & $33.61^{\mathrm{A}}$ & $14.13 \pm 7.98$ & $<0.01$ & $<0.01$ & $<0.01$
\end{tabular}

Vel - Velocidade; A - Arrasto; Pot - Potência; Int - Interação; Cond - Condição. Significativamente maior que a outra condição para o mesmo grupo; ${ }^{B}$ Significativamente maior que o outro grupo para a mesma condição.

Fonte: construção da autora 
Na comparação entre os grupos para a contribuição relativa de membros inferiores, foram encontrados significativamente maiores para o grupo recreativo $(p=0.02)$, sendo a contribuição relativa para este grupo de 33,9 $\pm 23,1 \%$, enquanto o grupo nacional apresentou uma contribuição relativa de membros inferiores de $22,5 \pm 10,3 \%$.

\section{DISCUSSÃO}

Como esperado os valores de todas as variáveis analisadas foi maior nas condições de nado completo do que nas condições de nado com membros inferiores apenas. Também era esperada a diferença entre os grupos competitivo e recreativo para ambas as condições, no entanto houve uma interação significativa entre estes fatores indicando que o acréscimo de velocidade pela inclusão das ações de membros superiores foi mais relevante para o grupo nacional. Este resultado pode ser devido a maior habilidade dos nadadores mais treinados em coordenar às ações de braços e pernas e se aproveitar da melhor maneira possível da interação entre elas (MEZÊNCIO et al., 2020).

Para os valores de arrasto não houve diferença entre os grupos para a condição de propulsão utilizando membros inferiores apenas. O arrasto na natação é normalmente modelado com uma função quadrática da velocidade (AMAR, 1920), desta forma, considerando a menor diferença de velocidade entre os grupos para esta condição, bem como o menor valor de velocidade quando comparada com a condição completa, este resultado era matematicamente esperado (KOLMOGOROV; DUPLISHCHEVA, 1992). Embora não tenha havido significância estatística, é interessante notar que o arrasto do grupo recreativo é ligeiramente superior ao grupo nacional. Este resultado seria esperado em teoria, pois, devido à menor habilidade deste grupo, eles poderiam apresentar uma maior dificuldade em vencer o torque subaquático, o que aumentaria a área de secção transversa exposta ao escoamento e consequentemente o arrasto ativo (ZAMPARO et al., 1996). Contudo, como o resultado não foi significativo é impossível afirmar que houve qualquer efeito determinístico como causa destes resultados.

Para potência também não houve diferença entre os grupos para a condição de nado com membros inferiores apenas. A potência é o produto entre o arrasto e a velocidade e, portanto, seu resultado é diretamente dependente destes. Por conseguinte, a ausência de 
diferença entre o arrasto para os grupos foi mais relevante nesta relação do que a diferença observada pela velocidade, e isso pode ser devido às mesmas observações mencionadas para o arrasto.

A contribuição relativa de membros inferiores para ambos os grupos mostrou valores condizentes com aqueles reportados na literatura (BUCHER, 1974; HOLLANDER et al., 1988; KARPOVICH, 1935). O grupo recreativo apresentou uma contribuição relativa de membros inferiores maior que o grupo nacional, e isto pode ter sido causado por dois fatores. Primeiramente, como mencionado anteriormente, nadadores mais habilidosos são capazes de sincronizar suas ações de pernas e braços de forma a produzir maior eficiência das ações propulsivas, o que consequentemente, reduziria a participação de membros inferiores apenas na potência total produzida (MEZÊNCIO et al., 2020). Além disso, a pernada não possui apenas finalidade propulsiva no nado crawl, mas também tem grande importância na estabilidade do corpo durante o nado (NAKASHIMA, 2007; YANAI, 2001; ZAMPARO et al., 1996), desta forma a maior contribuição de membros inferiores para o grupo de menor desempenho pode ser relacionada a uma maior necessidade de estabilização devido a uma técnica menos eficiente.

A principal limitação do estudo é a própria limitação do VPM. Os pressupostos do VPM são violados, o que prejudica a precisão dos dados, de toda forma esta violação não afeta a validade do método, ou seja, o fenômeno avaliado pelo VPM é realmente o arrasto ativo dos nadadores. Apesar desta limitação o VPM ainda é um dos principais métodos de medição do arrasto ativo devido à sua especificidade, é o método que avalia o arrasto em condições mais próximas das condições reais de nado (TOUSSAINT; ROOS; KOLMOGOROV, 2004). Além da maior especificidade a simplicidade de aplicação do método também favorece sua utilização na prática.

\section{CONCLUSÃO}

Os valores de contribuição relativa de membros inferiores avaliados pelo VPM foram condizentes com os previamente reportados na literatura e as variáveis analisadas apresentaram um comportamento entre os testes condizentes com aquele esperado para as características biomecânicas da tarefa. Desta forma o VPM se mostrou uma técnica de fácil execução capaz de avaliar a contribuição relativa de membros inferiores no nado crawl, tornando esta informação acessível para treinadores. 


\section{REFERÊNCIAS BIBLIOGRÁFICAS}

AMAR, Jules. The human motor. London, England: Routledge, 1920.

BUCHER, Walter. Influence of the leg kick and the arm stroke on the total speed during the crawl stroke. International symposium on biomechanics in swimming, 2. Anais... Brussels, BE: University Park Press, 1974.

COUNSILMAN, James. La natacion: ciencia y tecnica. Barcelona, España: Editorial Hispano Europea, 1971.

FINA. Fina Facilities 2016. Dules, Disponível em: <http://www.fina.org/sites/default/files/rules-print-pdf/8458.pdf> Acesso em: 23 de mai. de 2021.

HOLLANDER, Peter e colaboradores. Measurement of active drag during crawl arm stroke swimming. Journal of sports sciences, v. 4, n. 1, p. 21-30, mar., 1986.

HOLLANDER, Peter; DE GROOT, Gert; VAN INGEN SCHENAU, Gerrit Jan. Contribution of the legs to propulsion in front crawl swimming. Swimming science, v. 18, p. 39-43, 1988.

KARPOVICH, Peter. Analysis of the Propelling Force in the Crawl Stroke. Research quarterly, v. 6, n. 2, p. 49-58, 1935.

KOLMOGOROV, Sergei; DUPLISHCHEVA, O. Active drag, useful mechanical power output and hydrodynamic force coefficient in different swimming strokes at maximal velocity. Journal of biomechanics, v. 25, n. 3, p. 311-318, 1992.

MEZÊNCIO, Bruno e colaboradores. Overall indexes of coordination in front crawl swimming. Journal of sports sciences, v. 38, n. 8, p. 910-917, 2020.

NAKASHIMA, Motomu. Mechanical study of standard six beat front crawl swimming by using swimming human simulation model. Journal of fluid science and technology, v. 2, n. 1, p. 290-301, 2007.

YANAI, Toshimasa. Rotational effect of buoyancy in front crawl: does it really cause the legs to sink? Journal of biomechanics, v. 34, n. 2, p. 235-243, 2001.

ZAMPARO, Paola e colaboradores. Effect of the underwater torque on the energy cost, drag and efficiency of front crawl swimming. European journal of applied physiology and occupational physiology, v. 73, n. 3-4, p. 195-201, 1996.

\section{Dados da autora:}

Email: raisavalvassori@gmail.com 
Endereço: Escola de Educação Física e Esporte - Universidade de São Paulo (EEFE - USP), Avenida Professor Mello Moraes, 65, Vila Universitária, São Paulo, SP, CEP: 05508-030, Brasil.

Recebido em: 24/05/2021

Aprovado em: 02/06/2021

\section{Como citar este artigo:}

VALVASSORI, Raísa e colaboradores. A estimativa da contribuição de pernas no nado de crawl. Corpoconsciência, v. 25, n. 2, p. 123-133, mai./ ago., 2021. 